'K Mayes, ${ }^{2} \mathrm{M}$ Tan, ${ }^{3} \mathrm{C}$ Morgan. ${ }^{1} \mathrm{Clinical}$ Chemistry; ${ }^{2}$ Paediatrics, Alder Hey Children's Hospital; ' Neonatology; Liverpool Women's NHS Foundation Trust, Liverpool, UK

Background Hyperalimentation describes the increase in glucose, amino acid (AA) and lipid intake designed to overcome postnatal growth failure in preterm infants. We have previously shown increasing parenteral AA intake increased 14/22 individual AA levels with only tyrosine lower. Hyperalimentation increases hyperglycaemia requiring insulin treatment. We hypothesised insulin administration may increase AA utilisation so lowering AA levels.

Aim To compare the plasma AA profiles in preterm infants with insulin-treated hyperglycaemia with those whose did not receive insulin.

Methods Infants $<29$ weeks gestation were originally randomised to receive hyperalimentation ( $25 \%$ more glucose, $4 \mathrm{~g} / \mathrm{kg} /$ day versus $3 \mathrm{~g} / \mathrm{kg} /$ day protein/lipid) or a control regimen within 5 days of birth with head growth as the primary outcome. The study protocol recorded actual nutrient intake and parenteral nutrition "intolerance" including hyperglycaemia, insulin use and AA profiles. AA levels were measured on day 9 (ion exchange chromatography).

Results 118 AA profiles were obtained from 142 infants on day $8-10$. Secondary analysis restratified data to compare insulin $(n=57$; hyperalimentation $n=37$ ) with no insulin ( $n=61$; hyperalimentation $\mathrm{n}=20$ ) treatment. Infants receiving insulin were of lower gestation/ birthweight $(\mathrm{p}<0.01)$ and received more protein $(3.0 \mathrm{~g} / \mathrm{kg} /$ day versus $2.7 \mathrm{~g} / \mathrm{kg} /$ day; $\mathrm{p}=0.02$ ) mainly as intravenous $\mathrm{AA}$, when compared to those not receiving insulin. The insulin-treated group had lower levels in $9 / 22 \mathrm{AAs}(p<0.05)$ and no statistically significant difference in the remaining 13 ( $p>0.05)$

Conclusion Preterm infants with insulin-treated hyperglycaemia have lower AA levels on day 8-10 despite lower birthweight, gestation and higher protein intake. This suggests exogenous insulin may improve AA utilisation for protein synthesis.

\section{EVALUATION AND COMPARISON OF CALCIUM AND PHOSPHORUS IN THE IMPROVEMENT OF METABOLIC BONE DISORDER IN PREMATURE INFANTS}

doi:10.1136/archdischild-2012-302724.1354

Z Torabi, N Moemeni, S Mazloozadeh. Zanjan University of Medical Sciences, Zanjan, Iran

Background Metabolic bone disease is a common condition among premature Neonates. The aim of this study was to determine the impact of calcium and phosphorus on radiological and biochemical character osteopenia in premature neonates.

Methods This trial was done in forty premature Neonates over a period of six months in the All these babies are fed with breast milk, and 400 units of vitamin D daily They, randomly divided into two groups. Half of these babies received supplement of Calcium (45 $\mathrm{mg} / \mathrm{kg} /$ day) and phosphorus $(24 \mathrm{mg} / \mathrm{kg} /$ day $)$.

Serum calcium, phosphorus, and alkaline phosphatase with growth parameters (including weight, height, and head circumference) was measured every two weeks. At the end of this time wrist $\mathrm{x}$-ray for evaluating of osteopenia was done. The collected data was analyzed with SPSS 11.5.

Results Radiological changes characteristic of osteopenia have been found in $40 \%$ ( 8 cases) of infants in the case group and 65\% (13 cases) of infants in the control group $(\mathrm{P}=0.113)$. Serum calcium, phosphorus, and alkaline phosphatase levels was not statistically different $(\mathrm{P}>0.05)$. Weight gain was similar in both groups $(\mathrm{P}=0.097)$. but, linear and head circumference rise in the case group were significantly greater than control group ( $\mathrm{P}=0.002$ and $\mathrm{P}=0.015$, respectively).

Conclusion Calcium and phosphorus supplementation in preterm breast-fed infants were seem to be effective on prevention of osteopenia and improvement of growth. Thus, we recommend oral calcium and phosphate supplement addition accompanying with breast-feeding in premature neonate.

\section{INCIDENCE OF SERUM HYPOPHOSPHATEMIA IN GROWTH RESTRICTED AND APPROPRIATELY GROWN PRETERM INFANTS}

doi:10.1136/archdischild-2012-302724.1355

${ }^{1,2} \mathrm{~F}$ Moya, 'D Kurtz, ${ }^{1} \mathrm{JM}$ Oliver. ${ }^{1}$ Neonatology, Coastal Carolina Neonatology, Wilmington; ${ }^{2}$ Pediatrics, University of North Carolina, Chapel Hill, NC, USA

Background Infants with intrauterine growth restriction (IUGR) often have metabolic and electrolyte abnormalities. Our aim was to determine the incidence of hypophosphatemia in IUGR versus appropriate for gestational age (AGA) premature infants.

Methods A retrospective review of infants $\leq 32$ weeks or $\leq 1500$ grams who had a serum phosphorus within 48 hours after birth. We collected maternal and neonatal demographic data and electrolyte values. Infants below the 10th percentile on the Fenton Growth Curve were categorized as IUGR. Serum hypophosphatemia was defined as $<4 \mathrm{mg} / \mathrm{dL}$ and serum hypokalemia as $<3.5 \mathrm{mg} / \mathrm{dL}$.

Results Over a 4 year period, 304 infants were eligible. Of these, 54 were IUGR (mean birth weight (BW) of 848 grams and mean gestational age (GA) of $28+6$ weeks) and 250 were AGA (mean BW of 1067 grams and mean GA of $27+6$ weeks). $48 \%$ of the IUGR infants had hypophosphatemia compared with only $6 \%$ of the AGA infants $(p<0.05)$. The IUGR infants with hypophosphatemia had a lower birth weight and GA than the IUGR infants without hypophosphatemia. This difference was not observed among AGA infants. 15.1\% of the IUGR infants $(8 / 53)$ had a serum potassium of $<3.5 \mathrm{mg} / \mathrm{dL}$ compared to $7.6 \%$ of the AGA infants $(19 / 250)$. There was a moderate correlation between serum phosphorus and serum potassium. Overall mortality was $<1 \%$

Conclusions Hypophosphatemia is very common among IUGR infants $<32$ weeks GA and there is a moderate correlation with hypokalemia. These electrolyte abnormalities probably reflect adaptive mechanisms associated with growth restriction in utero.

\section{CONTINUOUS GLUCOSE MONITORING IN VERY LOW BIRTHWEIGHT PRETERM INFANTS ON FULL ENTERAL FEEDS}

doi:10.1136/archdischild-2012-302724.1356

'E Mola Riehle, ${ }^{2} \mathrm{~A}$ Staffler, ${ }^{1} \mathrm{M}$ Klemme, ${ }^{1} \mathrm{~A}$ Schulze, ${ }^{1} \mathrm{AW}$ Flemmer. 'Div. Neonatology, Perinatal Center, Ludwig-Maximilian-University Munich, Munich, Germany; ${ }^{2}$ Div. Neonatology, Regional Hospital Bolzano, Bolzano, Italy

Background We previously observed hypoglycaemic episodes in preterm infants after achieving full enteral feeds and during a stable postnatal period. The purpose of this study was to prospectively determine subcutaneous glucose levels in this population.

Methods Preterm infants $<32$ wks gestational age were enrolled for continuous subcutaneous glucose monitoring over $72 \mathrm{hrs}$ in two cohorts: A: $500-999 g(n=16) ;$ B: 1000-1500g $(n=9)$. All infants were fed according to a standard feeding protocol where full feeds are provided at $150-180 \mathrm{ml} / \mathrm{kg} / \mathrm{d}$ of fortified EBM or premature formula at $110-135 \mathrm{kcal} / \mathrm{kg} / \mathrm{d}$. Primary outcome was the frequency and quality of hypoglycaemic episodes within 72 hours, defined as tissue glucose $<2.5 \mathrm{mmol} / \mathrm{L}$.

Results $81.3 \%$ of the infants in A and $44.4 \%$ in B showed relevant glucose fluctuations during monitoring. Hypoglycaemic episodes occurred in $37.5 \%$ in group A, compared to $22.2 \%$ in group B. In group A $7 \%$ of infants showed glucose values below $1.7 \mathrm{mmol} / \mathrm{L}$. We also observed hyperglycaemic episodes $(>8.3 \mathrm{mmol} / \mathrm{L})$ after feeds $(\mathrm{A}$ : $57 \%$, B:17\%), followed by rapid drops in both cohorts. Cumulatively, all hypo- and hyperglycaemic episodes lasted $>60 \mathrm{~min}(16 \%)$, $35-60 \mathrm{~min}(21 \%), 10-30 \mathrm{~min}(60 \%)$ and $<5 \mathrm{~min}(3 \%)$ per patient. The main risk factors for glucose instability were gestational age and weight at trial. 
Conclusion Otherwise stable, well developing former very low birth weight preterm infants are at risk for glucose instability, generally considered as unfavourable. It remains unclear whether this instability is likely to influence long-term outcome and whether continuous feeds are preventive.

\section{SIGNIFICANCE OF HIGH ALKALINE PHOSPHATASE LEVELS WITH IN FIRST TWO WEEKS OF LIFE AMONG EXTREMELY PRE-TERM BABIES}

doi:10.1136/archdischild-2012-302724.1357

AK Gupta, H Ali. Neonatology, The Leeds Teaching Hospitals NHS Trust, Leeds, UK

Background Serum alkaline phosphatase levels (S-ALP) are often high among extremely preterm babies before first two weeks of life. It is not certain whether this represents increased physiological bone turn-over or is a predictor for osteopenia of prematurity.

Aim To study the relationship between osteopenia of prematurity and peak S-ALP levels with in first two weeks among pre-term babies born before twenty-nine weeks gestation.

Methods We evaluated seventy-three extremely pre-term babies born before twenty-nine weeks gestation who were admitted to tertiary neonatal units in Leeds, UK from 01/01/2009 to 31/01/2011. S-ALP, calcium and inorganic phosphate were checked regularly while they were in the neonatal unit. Forty out of seventy three babies had radiographs performed after five weeks post-natal age and were reported by radiologist.

Results In our cohort of seventy-three extremely pre-term babies, $55 \%$ had peak S-ALP levels exceeding $1200 \mathrm{iu} / 1$ (four times the upper limit of normal for adults) with in first two weeks. Infants who developed osteopenia had significantly lower gestational age and birth weight, and were significantly more likely to receive postnatal steroids. Radiologically proven osteopenia developed in $74 \%$ of infants with peak S-ALP exceeding $1200 \mathrm{iu} / 1$ compared to $35 \%$ of infants with peak S-ALP below $1200 \mathrm{iu} / 1$ before two weeks postnatal age ( $p$-value 0.014).

Conclusion S-ALP exceeding $1200 \mathrm{iu} / \mathrm{l}$ with in two weeks postnatal age is associated with 2.1 fold increased risk of development of osteopenia in extremely pre-term infants.

\section{EFFECT OF MODE OF DELIVERY ON MORTALITY AND MORBIDITY IN VERY LOW BIRTH WEIGHT NEONATES WITH RESPIRATORY DISTRESS SYNDROME}

doi:10.1136/archdischild-2012-302724.1358

A Dursun, BS Karagol, N Hakan, N Karadag, A Zenciroglu, N Okumus, D Dilli, S Beken. Neonatology, Dr Sami Ulus Maternity and Children's Health and Diseases Training and Research Hospital, Ankara, Turkey

Background \& Aim: It is known that wet lung syndrome and pulmonary maladaptation are more frequent in infants delivered by cesarean section while the effect of mode of delivery on RDS is unknown. In this study, we analyzed the effect of the mode of delivery on RDS outcome in very low birth weight (VLBW) neonates.

Methods Data of all the VLBW neonates with respiratory distress syndrome (RDS) between 2007 and 2012 was retrospectively analyzed. Gestational age, gender, birth weight, mode of delivery, necrotizing enterocolitis (NEC), intracranial bleeding (ICH), patent ductus arteriosus (PDA), retinopaty of prematurity (ROP), broncopulmonary dysplasia (BPD) were noted.

Results A total of 186 newborns were diagnosed as VLBW neonates with RDS among the 5980 neonates hospitalized in NICU. Mean birth weight and gestational age were $1058 \pm 261$ grams and $28 \pm 2.7$ weeks, respectively. Cesarean delivery rate was $62.7 \%$. There were no differences with respect to birth weight, gestational age and gender between mode of delivery. There was no significant relationship between the NEC, PDA, ROP, BPD and mode of delivery. Also no significant relationship between the mode of delivery and mortality was determined. On the other hand, ICH was significantly higher in neonates delivered vaginally ( $48 \%$ vs. $31 \%$, p $<0.05$ ).

Conclusion Mode of delivery has no effect on the mortality and morbidity of RDS in VLBW neonates but ICH was significantly higher in normal vaginal delivery group. Therefore, mode of delivery should be decided on the basis of obstetrical indications.

\section{NEONATAL MORTALITY AND MORBIDITY IN DIFFERENT SOCIOECONOMIC CLASSES IN SOUTHWEST OF IRAN}

doi:10.1136/archdischild-2012-302724.1359

N Maharlouei, KB Lankarani. Health Policy Research Center, School of Medicine, Shiraz University of Medical Sciences, Shiraz, Iran

Background and Aims Socioeconomic inequality in infant mortality and morbidity are challenging subjects even in many developed countries. In this study we compared neonatal mortality and morbidity in different socioeconomic status (SES) in Fars, Iran.

Methods A cross-sectional study was conducted in Fars, the fifth populated province in Iran from March to October, 2011. Using cluster random sampling method, data was collected by interviewing mothers two months after delivery and filling the check list from their health file. We categorized interviewees into low, middle and high SES according to their education, job, and wealth.

Results 2106 (93.6\%) mothers participate in this study. Of them $11(0.9 \%)$ lost their fetus in pregnancy, 8 mothers $(0.4 \%)$ experienced still birth while 18 mothers (0.8\%) lost their baby in neonate period. $97.3 \%$ of mothers gave birth in the hospital not related to their socioeconomic ranks $(\mathrm{P}=0.1)$. Also, no association was found between SES and APGAR ( $\mathrm{P}=0.06)$, frequency of fetal and neonatal dead $(p=0.1)$, and admission in neonatal intensive care units $(\mathrm{P}=0.2)$. Additionally, frequency of birth trauma (fracture of humorous, clavicle, femur and skull) did not statistically differ in these groups. However, congenital anomaly $(\mathrm{P}=0.005)$, icterus $(\mathrm{P}=0.004)$, neonatal convulsion $(\mathrm{P}=0.003)$ and neonatal infection $(\mathrm{P}=0.007)$ were highest in middle socioeconomic and lowest in wealthy group. Conclusions This study showed good access to health facilities irrespective of SES. More attention should be paid to neonates of middle SES group, since they suffered the most from neonatal morbidity.

\section{MECONIUM/STOOL AND URINARY PATTERNS OF HEALTHY TURKISH NEWBORNS}

doi:10.1136/archdischild-2012-302724.1360

${ }^{1} S M$ Kayıran, ${ }^{2} E$ Eroğlu, ${ }^{3} \mathrm{PG}$ Kayıran, ${ }^{4} \mathrm{~S}$ Sazak, ${ }^{5} \mathrm{~B}$ Gurakan. 'Deapartment of Pediatrics; ${ }^{2}$ Department of Pediatric Surgery; ${ }^{3}$ Department of Pediatrics, American Hospital; " ${ }^{4}$ Deapartment of Pediatrics, Ministry of Health, Okmeydani Training Hospital; ${ }^{5}$ Department of Pediatrics, Division of Neonatology, American Hospital, Istanbul, Turkey

Background and Aims Routine follow-ups of healthy newborns for up to $48 \mathrm{~h}$ for those delivered by normal vaginal delivery (NVD) and for up to $96 \mathrm{~h}$ for those delivered by cesarean section (CS) are highly recommended as they facilitate the early detection of certain problems.

Objectives To investigate the meconium/stool and urinary patterns of healthy Turkish neonates, and to determine whether they correlate with delivery mode, birth weight and feeding method and frequency.

Methods Newborns with a gestational age of $\geq 34$ wk were included. The frequency of meconium/stool and urine passage and the delivery mode, birth weight and feeding method and frequency were recorded throughout their hospital stay. 